\title{
Depletion of RhoGDI2 expression inhibits the ability of invasion and migration in pancreatic carcinoma
}

\author{
${\text { BIN } \mathrm{YI}^{1 *}, \text { YOU HU }}^{1 *}$, GONGZHAO QIN ${ }^{2 *}$, WEN GU $^{1}$, XINGUO ZHU $^{1}$, \\ SONGBING HE ${ }^{1,3}$, JIAN ZHOU ${ }^{1}$ and DECHUN LI ${ }^{1}$ \\ ${ }^{1}$ Department of General Surgery, The First Affiliated Hospital of Soochow University, Suzhou, Jiangsu 215006; \\ ${ }^{2}$ Department of Gynaecology and Obstetrics, Suzhou Municipal Hospital, Suzhou, Jiangsu 215003; \\ ${ }^{3}$ Shanghai Institute of Immunology, Shanghai Jiao Tong University School of Medicine, Wan Qu, Shanghai 200025, P.R. China
}

Received December 21, 2013; Accepted April 24, 2014

DOI: $10.3892 /$ ijmm.2014.1765

\begin{abstract}
Rho GDP dissociation inhibitor 2 (RhoGDI2) has been identified as a regulator of tumor metastasis, although its role in tumor progression remains controversial. In this study, we examined the expression of RhoGDI2 in PC tissues and cell lines. To investigate the function of RhoGDI2 in PC cells, RhoGDI2 expression was depleted in PANC-1 and Patu8988 cells by small interfering RNA (siRNA). RhoGDI2 was found to be overexpressed in pancreatic carcinoma (PC) tissues and PC cell lines. Additionally, the results showed that depletion of RhoGDI2 significantly inhibited cell motility and invasion in vitro, but did not affect cell proliferation. The clinical study together with the experimental data confirmed that RhoGDI2 modulated the expression of matrix metalloproteinase 2 (MMP2). Taken together, findings of the present study indicated that RhoGDI2 is involved in pancreatic tumor malignancy and metastasis. Thus, RhoGDI2 is a potential target for the gene therapy of PC.
\end{abstract}

\section{Introduction}

Pancreatic carcinoma (PC) is an aggressive and lethal malignancy. The median overall survival is $<6$ months, with a 5-year survival rate of $5 \%(1,2)$. Vascular invasion, perineural invasion and distant metastasis are the critical features in the aggressive phenotype of PC, and contribute to the lost opportunities for surgical resection $(3,4)$. Thus, new therapeutic strategies

Correspondence to: Dr Dechun Li or Dr Jian Zhou, Department of General Surgery, The First Affiliated Hospital of Soochow University, 188 Shizi Street, Suzhou, Jiangsu 215006, P.R. China E-mail: dechunlisuzhou@gmail.com

E-mail: zhoujian0612@gmail.com

${ }^{*}$ Contributed equally

Key words: Rho GDP dissociation inhibitor 2, pancreatic carcinoma, matrix metalloproteinase 2 , invasion, metastasis should be based on a better understanding of biomarkers and their association with invasion and metastasis.

Rho GTPases, including Rac1, Cdc42 and RhoC, are important regulators of cell migration, cell motility, cell cycle progression and cytoskeleton organization $(5,6)$. The biological activities of Rho GTPases are regulated by guanine nucleotide exchange factors (GEFs), GTPase-activating proteins (GAPs) and Rho GDP dissociation inhibitors (RhoGDIs) (7). Rho GDP dissociation inhibitor 2 (RhoGDI2), also known as D4-GDI or LyGDI, belongs to a family of RhoGDIs. RhoGDI2 was originally expressed in hematopoietic cells, predominantly in $\mathrm{B}$ and T cells $(8,9)$. However, it has been shown that RhoGDI2 is also abnormally expressed in tumors. The role of RhoGDI2 in tumor progression remains controversial. RhoGDI2 may function as a positive regulator of tumor progression in gastric, colorectal, and breast cancer (10-12). The role of RhoGDI2 as a metastasis suppressor gene was also validated in bladder cancer and Hodgkin's lymphoma $(13,14)$. However, the expression and role of RhoGDI 2 in the progression of $\mathrm{PC}$ remains to be determined.

In this study, we examined the expression of RhoGDI2 in PC tissues and cell lines. Moreover, using small interfering RNA (siRNA) to silence RhoGDI2 expression, we investigated the effect of RhoGDI2 in the regulation of invasion and metastasis. These findings indicated that RhoGDI2 is a potential target for the gene therapy of PC.

\section{Materials and methods}

Clinical samples. Tissue samples from $60 \mathrm{PC}$ patients were collected during surgical resections performed at the First Affiliated Hospital of Soochow University between January 2010 and December 2012. Tumorous and adjacent non-tumorous tissues were frozen immediately after surgical removal in liquid nitrogen and stored at $-80^{\circ} \mathrm{C}$. The patients did not receive any preoperative chemotherapy, radiotherapy or immunotherapy. The samples were obtained following written patient consent. Study approval was obtained from the local ethics committee of Soochow University.

Cell culture and transfection. Human PC cell lines, PANC-1, SW1990, Patu8988 and BxPC-3 were obtained from the 
Shanghai Institute of Biochemistry and Cell Biology (Shanghai, China) and maintained in DMEM, supplemented with $10 \%$ fetal bovine serum (both from Gibco, Grand Island, NY, USA) and $100 \mu \mathrm{g} / \mathrm{ml}$ each of penicillin and streptomycin (Invitrogen, Carlsbad, CA, USA) in $5 \% \mathrm{CO}_{2}$ at $37^{\circ} \mathrm{C}$.

SiRNA transfection. Small hairpin RNA (shRNA) of human RhoGDI2 (NM_001175; GeneBank) transfer vector encoding the green flourescent protein (GFP) was constructed by GeneChem Co., Ltd. (Shanghai, China). The siRNA sequence targeting RhoGDI2 (siRNA-RhoGDI2) was 5'-GGAAGGUUC UGAAUAUAGA-3', as confirmed by sequencing. The negative control of siRNA (siRNA-NC) was designed and the sequence was without obvious homology to the human gene. Cells $\left(1 \times 10^{4} /\right.$ well) were cultured in 6-well plates overnight and reached $60-70 \%$ confluence. To determine the different expressions of RhoGDI2, PANC-1 and Patu8988 cells were transfected with siRNA-RhoGDI2 or siRNA-NC using Lipofectamine 2000 (Invitrogen) as the transfection reagent. Non-transfected cells were used as the blank control (control).

Quantitative PCR ( $q P C R)$. Total RNA from tissues and cells was extracted using TRIzol (Invitrogen). Single-strand cDNA for a PCR template was synthesized from $10 \mu \mathrm{g}$ of total RNA using random primers and M-MLV reverse transcriptase (Takara Bio, Dalian, China). The relative levels of target gene mRNA transcripts to that of the control ( $\beta$-actin) were determined by qPCR. The primers used were (forward and reverse): 5'-ATGACTGAAAAAGCCCCA-3' and 5'-TCATTC TGTCCACTCCTT-3' for RhoGDI2 (606bp); 5'-GTGCTGAAG GACACACTAAAGAAGA-3' and 5'-TTGCCATCCTTCTCA AAGTTGTAGG-3' for matrix metalloproteinase 2 (MMP2) (605 bp); 5'-AGCGGGAAATCGTGCGTG-3' and 5'-CAGGGT ACATGGTGGTGCTGCC- $3^{\prime}$ for $\beta$-actin (308 bp). The PCR reactions were 40 cycles $\left(95^{\circ} \mathrm{C}\right.$ for $15 \mathrm{sec}, 62^{\circ} \mathrm{C}$ for $45 \mathrm{sec}$, and $72^{\circ} \mathrm{C}$ for $30 \mathrm{sec}$ ). The amplified segments were analyzed by $2.5 \%$ agarose gels.

Western blotting. Cells were collected and lysed in lysis buffer on ice. Total proteins were separated by $10 \%$ SDS-PAGE and blotted on PVDF membrane. Membranes were blocked with $10 \%$ non-fat milk powder at room temperature for $2 \mathrm{~h}$ and incubated with primary antibodies: anti-RhoGDI2 antibody (1:200), anti-MMP2 antibody (1:200) (both from Abcam, Cambridge, UK) and anti- $\beta$-actin antibody (1:200; Santa Cruz Biotechnology, Inc., Santa Cruz, CA) at $4^{\circ} \mathrm{C}$ overnight. After three washes, the membranes were incubated with a horseradish peroxidase-conjugated goat anti-mouse $\operatorname{IgG}(1: 2,000$; Santa Cruz Biotechnology, Inc.) for $2 \mathrm{~h}$ at room temperature. Reactive bands were detected using ECL western blotting detection reagent.

Cell proliferation assay. CCK8 assay was performed to evaluate cell proliferation. Following transfection, the cells were seeded in 96-well plates at $5 \times 10^{3}$ cells/well, followed by the addition of $20 \mu \mathrm{l} \mathrm{CCK8} \mathrm{(Dojindo} \mathrm{Laboratories,} \mathrm{Kumamoto,} \mathrm{Japan)} \mathrm{and}$ incubation at $37^{\circ} \mathrm{C}$ for an additional $2 \mathrm{~h}$. An ultraviolet spectrophotometer (Implen, Munich, Germany) was used to measure the absorbance of each well at $450 \mathrm{~nm}$, and each experiment was performed in triplicate and repeated five times.
In vitro wound scrape assay. Cells from each group were incubated in 6-well plates. A small wound area was made in the confluent monolayer with a $200 \mu 1$ pipette tip in a lengthwise stripe. The cells were washed twice with PBS and incubated at $37^{\circ} \mathrm{C}$. Images were captured at 0 and $48 \mathrm{~h}$. Wound width was measured at a magnification of x100 using a microscope (DM2500, Leica Microsystems, Mannheim, Germany) with a calibrated eyepiece grid $(1 \mathrm{~mm} / 100 \mu \mathrm{m}$ graduation). Ten measurements were determined at random intervals along the wound length.

Invasion assay. Cell invasion was determined using Matrigel-coated invasion Boyden chambers using a Transwell kit, according to the manufacturer's instructions. Briefly, $600 \mu \mathrm{l}$ DMEM medium containing 10\% FBS was added to the bottom chamber. After $12 \mathrm{~h}$ of serum starvation, the cells were collected and placed in the upper invasion chamber ( $1 \times 10^{5}$ cells/well). After incubation at $37^{\circ} \mathrm{C}$ for $48 \mathrm{~h}$, the non-invasive cells in the upper chamber were removed with a cotton swab. The insert membranes were fixed with methanol for $15 \mathrm{~min}$ and stained with $0.1 \%$ crystal violet. The stained cells that penetrated through the Matrigel were counted under the inverted microscope.

Immunohistochemistry (IHC). Serial sections $(4 \mu \mathrm{m})$ were prepared for immunohistological staining. Tissue sections were quenched for endogenous peroxidase with freshly prepared $3 \%$ $\mathrm{H}_{2} \mathrm{O}_{2}$ with $0.1 \%$ sodium azide and then placed in an antigen retrieval solution for $15 \mathrm{~min}$. After incubation in a casein block, primary anti-RhoGDI2 monoclonal antibody (1:50; Abcam) was applied to the sections for $1 \mathrm{~h}$ at room temperature, followed by incubation with the secondary antibody and extravidin-conjugated horseradish peroxidase. The staining intensity was scored as 0 (negative), 1 (weak), 2 (medium) and 3 (strong). The extent of staining was scored as $0(0 \%)$, $1(1-25 \%), 2(26-50 \%), 3(51-75 \%)$ and $4(>76 \%)$. The final score was obtained by the sum of the intensity score and the quantity score. A score $\geq 3$ was considered as a positive expression.

Statistical analysis. SPSS version 17.0 was used for statistical analysis. Data were expressed as mean $\pm \mathrm{SD}$. One-way analysis of variance (one-way ANOVA), t-test and Chi-square test were performed for inter-group comparison. $\mathrm{P}<0.05$ was considered statistically significant.

\section{Results}

Overexpression of RhoGDI2 protein in $P C$. In 60 patients who recently underwent surgery for PC, paired tumor samples and non-tumorous tissues were subjected to IHC. The subcellular expression pattern of RhoGDI2 was mainly diffuse cytoplasmic in PC and the non-tumorous tissues were negative or weekly positive for RhoGDI2 (Fig. 1). The positive rate of RhoGDI2 in tumor tissues was $73.3 \%$ (44/60) while that in non-tumorous tissues was $41.7 \%$ (25/60), showing a significant difference $\left(\chi^{2}=12.310, P=0.001\right)$.

Correlation between RhoGDI2 protein and clinicopathological characteristics. The relationship between the expression of RhoGDI2 and the clinicopathological characteristics of PC are shown in Table I. RhoGDI2 expression was markedly 


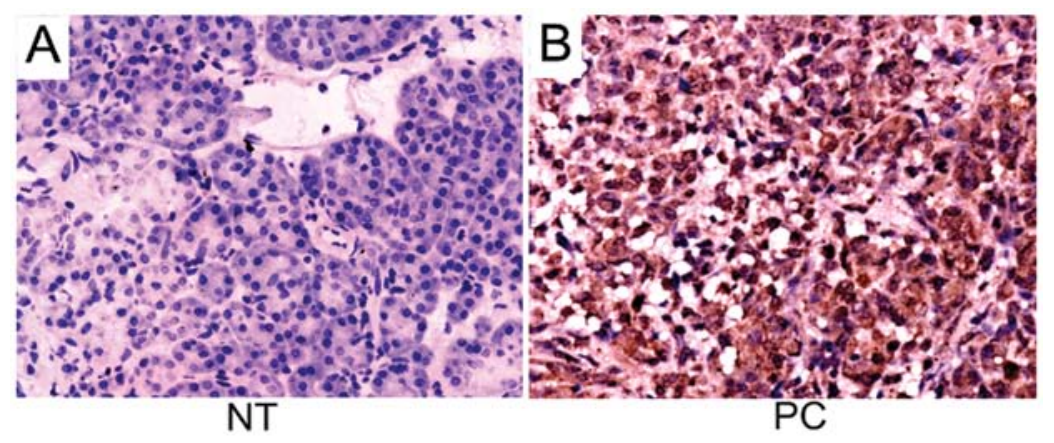

Figure 1. Immunohistochemical staining of Rho GDP dissociation inhibitor 2 (RhoGDI2) protein. (A) Low expression in pancreatic non-tumorous tissues (NT). (B) High expression in pancreatic carcinoma (PC) tissues. Original magnification, x200.

Table I. Relationship between RhoGDI2 expression and clinicopathological characteristics of PC patients.

\begin{tabular}{|c|c|c|c|c|c|}
\hline \multirow[b]{2}{*}{ Characteristics } & \multirow[b]{2}{*}{ Cases } & \multicolumn{2}{|c|}{ RhoGDI2 } & \multirow[b]{2}{*}{$\chi^{2}$} & \multirow[b]{2}{*}{ P-value } \\
\hline & & Negative & Positive & & \\
\hline \multicolumn{6}{|l|}{ Gender } \\
\hline Male & 38 & 10 & 28 & \multirow[t]{2}{*}{0.007} & \multirow[t]{2}{*}{1.000} \\
\hline Female & 22 & 6 & 16 & & \\
\hline \multicolumn{6}{|l|}{ Age (years) } \\
\hline$\leq 65$ & 36 & 13 & 23 & \multirow[t]{2}{*}{4.105} & \multirow[t]{2}{*}{0.072} \\
\hline$>65$ & 24 & 3 & 21 & & \\
\hline \multicolumn{6}{|l|}{ Tumor location } \\
\hline Head & 32 & 7 & 25 & \multirow[t]{2}{*}{4.105} & \multirow[t]{2}{*}{0.072} \\
\hline Body and tail & 28 & 9 & 19 & & \\
\hline \multicolumn{6}{|l|}{ Tumor size $(\mathrm{cm})$} \\
\hline$\leq 2$ & 8 & 6 & 2 & \multirow[t]{2}{*}{11.027} & \multirow[t]{2}{*}{$0.003^{\mathrm{a}}$} \\
\hline$>2$ & 52 & 10 & 42 & & \\
\hline \multicolumn{6}{|l|}{ Differentiation } \\
\hline Well & 13 & 6 & 7 & \multirow[t]{3}{*}{7.172} & \multirow[t]{3}{*}{$0.028^{\mathrm{a}}$} \\
\hline Moderate & 15 & 6 & 9 & & \\
\hline Poor & 32 & 4 & 28 & & \\
\hline \multicolumn{6}{|l|}{ Clinical stage } \\
\hline I & 12 & 8 & 4 & \multirow[t]{2}{*}{12.273} & \multirow[t]{2}{*}{$0.001^{\mathrm{a}}$} \\
\hline II & 48 & 8 & 40 & & \\
\hline \multicolumn{6}{|c|}{ Lymph node metastasis } \\
\hline $\mathrm{Y}$ & 41 & 6 & 35 & \multirow[t]{2}{*}{9.586} & \multirow[t]{2}{*}{$0.004^{\mathrm{a}}$} \\
\hline $\mathrm{N}$ & 19 & 10 & 9 & & \\
\hline \multicolumn{6}{|c|}{ Vascular invasion } \\
\hline $\mathrm{Y}$ & 18 & 1 & 17 & \multirow[t]{2}{*}{5.860} & \multirow[t]{2}{*}{$0.023^{\mathrm{a}}$} \\
\hline $\mathrm{N}$ & 42 & 15 & 27 & & \\
\hline
\end{tabular}

RhoGDI2, Rho GDP dissociation inhibitor 2; ${ }^{a} \mathrm{P}<0.05$.

correlated with tumor size $\left(\chi^{2}=11.027, \mathrm{P}=0.003\right)$, differentiation $\left(\chi^{2}=7.172, \mathrm{P}=0.028\right)$, clinical stage $\left(\chi^{2}=12.273, \mathrm{P}=0.001\right)$, lymph node metastasis $\left(\chi^{2}=9.586, \mathrm{P}=0.004\right)$ and vascular invasion $\left(\chi^{2}=5.860, \mathrm{P}=0.023\right)$, but did not show a statistically significant association with gender $\left(\chi^{2}=0.007, \mathrm{P}=1.000\right)$, age $\left(\chi^{2}=4.105, \mathrm{P}=0.072\right)$ and tumor location $\left(\chi^{2}=4.105, \mathrm{P}=0.072\right)$.
Depletion of RhoGDI2 by siRNA in PC cells. First, we examined the relative expression of RhoGDI2 at the mRNA and protein levels in the PANC-1, SW1990, Patu8988 and BxPC-3 cell lines, with PANC-1 and Patu8988 cells being selected as a high expression of RhoGDI2 (Fig. 2A and B). To investigate the function of RhoGDI2 in PC cells, we depleted RhoGDI2 
A

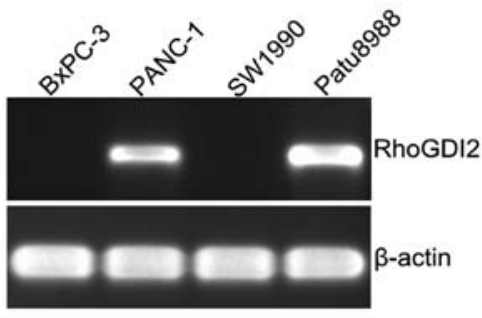

B

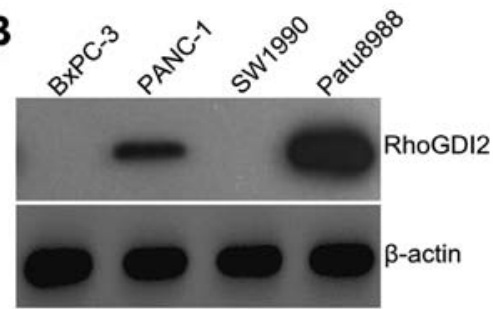

\section{C}

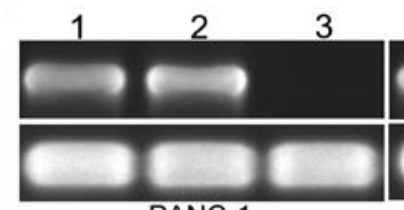

PANC-1

D

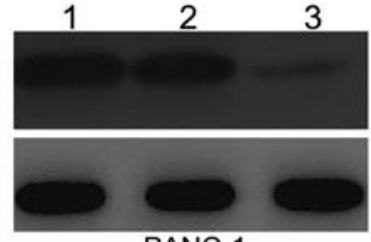

PANC-1

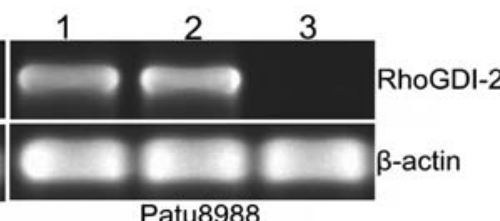

Patu8988

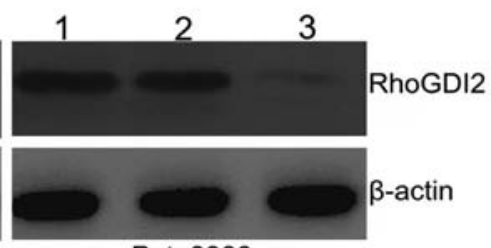

Patu8988

Figure 2. Silencing effect of Rho GDP dissociation inhibitor 2 (RHoGDI2). (A) Relative expression of RhoGDI2 mRNA in four PC cell lines (PANC-1, SW1990, Patu8988, BxPC-3) by quantitative PCR (qPCR). (B) Relative expression of RhoGDI2 protein in PC cell lines was assessed using western blotting. (C) The knockdown effect of RhoGDI2 mRNA was measured by qPCR. (D) The knockdown effect of RhoGDI2 protein was measured by western blotting. Lanes: 1, control; 2, RNAi-NC; and 3, RNAi-RhoGDI2.
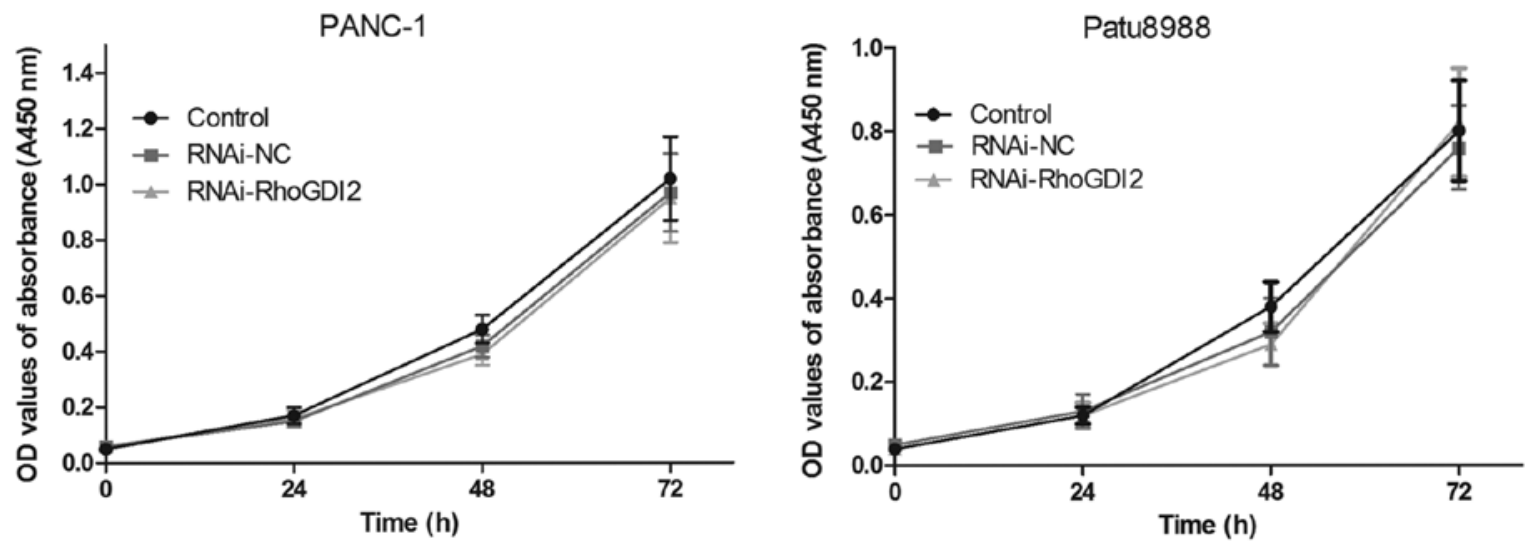

Figure 3. Cell proliferation following transfection with RNAi-RhoGDI2 or RNAi-negative control (NC) as analyzed by CCK8 assay. Data are the means \pm SD of five experiments.

expression in PANC-1 and Patu8988 cells using the RNAi method. As shown in Fig. 2C, the expression of RhoGDI2 mRNA was markedly decreased in the RNAi-RhoGDI2 group compared with the RNAi-NC group in PANC-1 and Patu8988 cells (both $\mathrm{P}<0.01$ ). A similar decrease was found at the protein level by western blotting (Fig. 2D), and the mean inhibition rate (the RNAi-RhoGDI2 group vs. the RNAi-NC group) was $78.3 \%$ in PANC-1 and $82.4 \%$ in Patu8988 (both $\mathrm{P}<0.05)$. These findings indicated that downregulation of the RhoGDI2 gene was specific and efficient.

Silencing RhoGDI2 inhibits invasion in PC cells. Using the transfected cells, CCK8 assay was performed to examine the effect of RhoGDI2 on PC cell proliferation. As shown in Fig. 3, there was no statistical significance in cell proliferation between the RNAi-RhoGDI2 and RNAi-NC groups $(\mathrm{P}>0.05)$.

The effect of RhoGDI2 on cell motility and invasion was assessed using wound scrape and Transwell assays. The wound scrape assay was used to evaluate the effect of RhoGDI2 depletion on cell motility in PANC-1 and Patu8988 cells. Time course analysis of the wound closure showed that the re-established period of the monolayer was significantly shorter in the RNAi-NC group than that in the RNAi-RhoGDI2 group (both $\mathrm{P}<0.05$ ) (Fig. 4). Invasion assays were performed to determine whether RhoGDI2 is required for PC cell invasion 

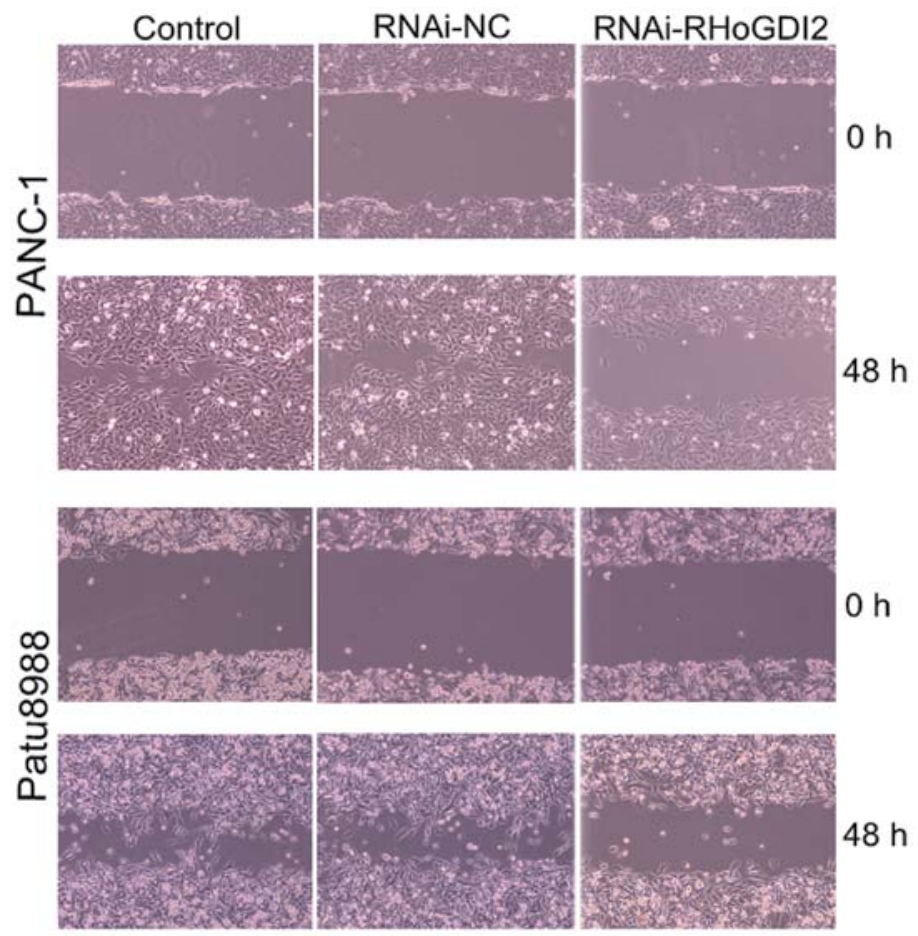

Figure 4. Cell migration as detected by the wound scrape assay. Monolayer images of cell migration in the wound scrape model at 0 and $48 \mathrm{~h}$ are shown; original magnification, $\mathrm{x} 100$.

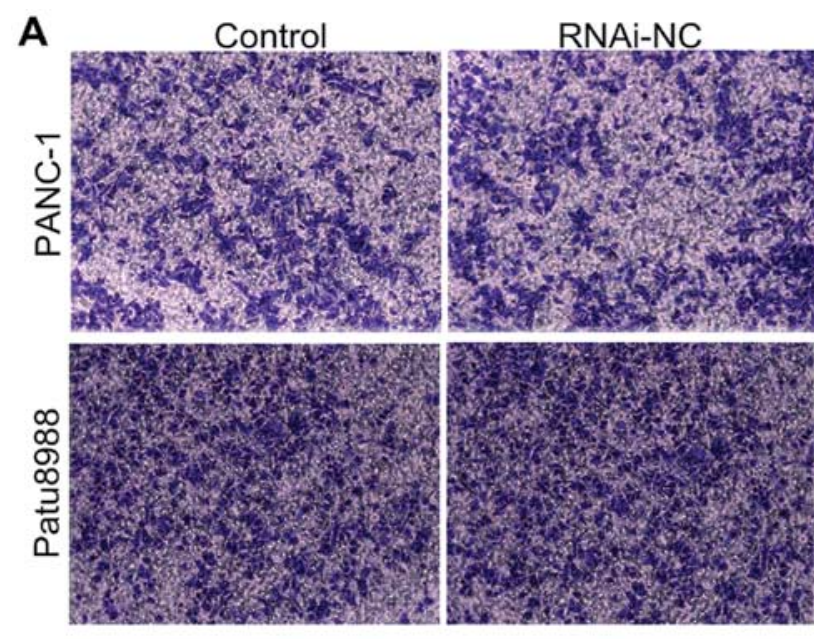

B

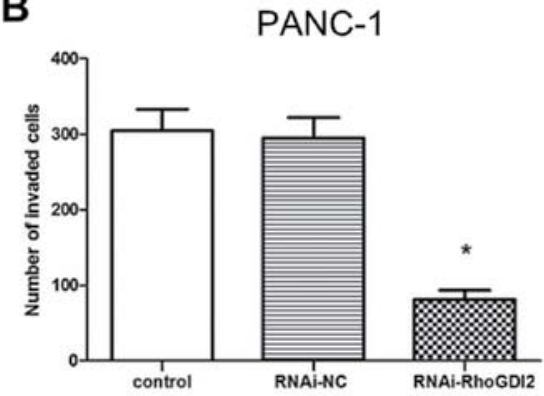

RNAi-RhoGDI2
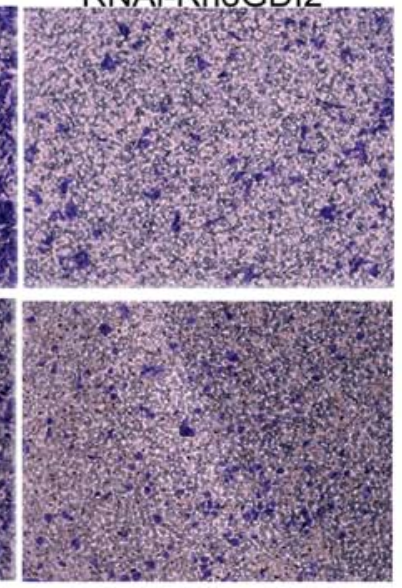

Patu8988

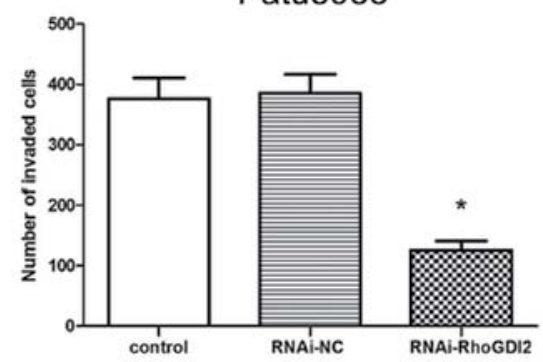

Figure 5. Cell invasion as detected by the Transwell assay. (A) Representative images of cell invasion captured under an inverted microscope (original magnification, $\mathrm{x} 100$ ). (B) The number of invaded cells in PANC-1 and Patu8988 cells. ("P<0.05).

based on the Boyden chamber assay. As shown in Fig. 5, depletion of RhoGDI2 significantly reduced cell invasion in the RNAi-RhoGDI2 group $(81 \pm 12$ cells per field for PANC- 1 and
$126 \pm 15$ cells per field for Patu8988) compared to the RNAi-NC group $(295 \pm 27$ cells per field for PANC-1 and $386 \pm 31$ cells per field for Patu8988) (both $\mathrm{P}<0.05$ ). 


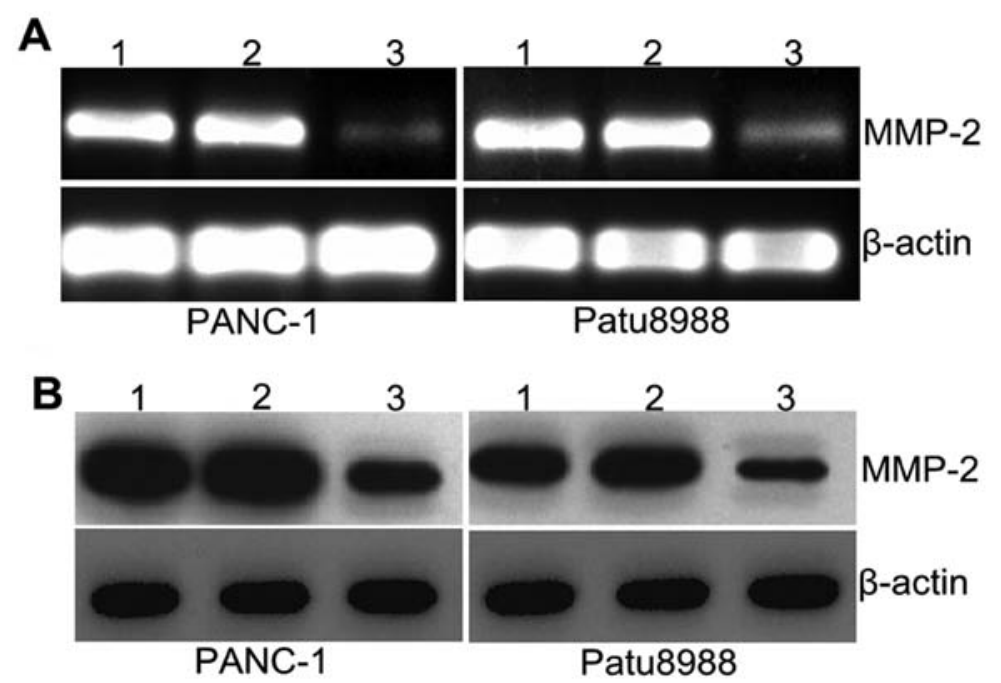

Figure 6. Silencing of Rho GDP dissociation inhibitor 2 (RhoGDI2) expression modulated the expression of matrix metalloproteinase 2 (MMP2) in pancreatic carcinoma (PC) cells as assessed by (A) qPCR and (B) western blotting. Lanes: 1, control; 2, RNAi-NC; and 3, RNAi-RhoGDI2.

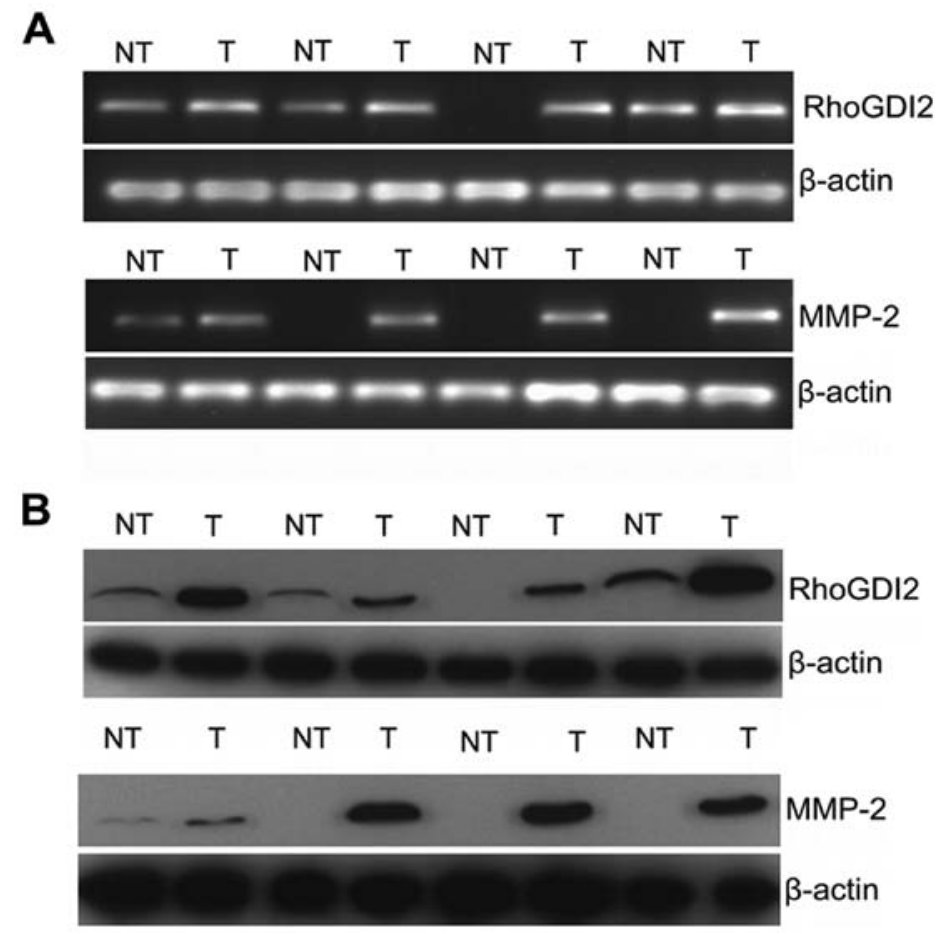

Figure 7. Correlation of expression of Rho GDP dissociation inhibitor 2 (RhoGDI2) and matrix metalloproteinase 2 (MMP2) in pancreatic carcinoma (PC). (A) Expression of RhoGDI2 and MMP2 mRNA was examined in PC and non-tumorous tissue (NT) by RT-PCR. (B) Expression of RhoGDI2 and MMP2 protein was examined in PC and NT by western blotting.

Silencing RhoGDI2 affects MMP2 expression. MMPs are involved in the degradation of extracellular matrix (ECM) and basement membranes. To determine whether RhoGDI2 promoted cancer cell invasion through MMPs, the expression of MMP2 was determined by qPCR and western blotting. Fig. 6A shows that MMP2 mRNA expression was significantly decreased in the RNAi-RhoGDI2 group compared with the RNAi-NC group in PANC-1 and Patu 8988 cells (both $\mathrm{P}<0.05$ ). Results of western blotting revealed a similar decrease at the protein level (both $\mathrm{P}<0.05$ ) (Fig. 6A). The mean inhibition rate was $62.6 \%$ vs. the RNAi-NC group in PANC-1 and $60.7 \%$ in
Patu8988. These results indicated that RhoGDI2 contributed to the expression of MMP2 in PC cells.

RhoGDI2 is positively correlated with MMP2 in PC. To obtain clinical evidence of a positive correction between RhoGDI2 and MMP2, the expression of 30 pairs of fresh PC and NT tissues were assessed by qPCR (Fig. 7A) and western blotting (Fig. 7B). The majority of PC exhibited overexpression of RhoGDI 2 compared with NT at the mRNA and protein levels (both $\mathrm{P}<0.01$ ). Similarly, MMP2 was overexpressed in PC compared with NT at the mRNA and protein level (both 
$\mathrm{P}<0.01)$. Results of subsequent analysis revealed that RhoGDI2 expression was positively correlated with MMP2 expression at the mRNA level (Spearman analysis, $\mathrm{r}=0.627, \mathrm{P}<0.001$ ). Consistent results were obtained when we compared the data at the protein level (Spearman analysis, $\mathrm{r}=0.817, \mathrm{P}<0.001$ ). Our clinical study together with the experimental data confirmed that RhoGDI2 modulated the expression of MMP2.

\section{Discussion}

In this study, the expression of RhoGDI2 in four PC cell lines, 30 matched clinical fresh $\mathrm{PC}$ tissues and 60 pairs of clinical paraffin-embedded PC with clinical data was identified. We found a tendency towards the upregulation of RhoGDI 2 in PC tissues compared to adjacent normal ones at the mRNA and protein levels. IHC results showed that the expression of RhoGDI2 was positively correlated with tumor size, clinical stage, lymph node metastasis and vascular invasion. In addition, depletion of RhoGDI2 in PANC-1 and Patu8988 cells by RNAi significantly inhibited cell motility and invasion in vitro, but had no effect on cell proliferation.

Three human RhoGDIs have been identified thus far: RhoGDI1, RhoGDI2 and RhoGDI3 (15-17). The proteins are key regulators of Rho GTPases function, such as cell motility, polarity and invasion. In contrast to RhoGDI1 and RhoGDI3, which are expressed ubiquitously, RhoGDI2 was reported originally to be expressed in hematopoietic cells, predominantly in B and T cells $(18,19)$. However, accumulating evidence shows that RhoGDI2 is also differentially expressed in human cancers (20). In the majority of studies, RhoGDI2 has been shown to promote tumor cell invasion, angiogenesis and metastasis, such as in lung and gastric cancer $(21,22)$. However, it can function as a metastasis suppressor gene in bladder cancer and Hodgkin's lymphoma (14,23). Our results showed that RhoGDI2 was overexpressed in PC and silencing RhoGDI2 inhibited cell invasiveness in PC cells. Those studies along with our findings indicate that the function of RhoGDI2 in cancer progression depends on different tumor types.

Although the reason for this discrepancy currently remains unclear, the conflicting role of RhoGDI2 may result from the dual roles of RhoGDI2 in the regulation of activities of Rho GTPases during cancer progression. RhoGDI2 bound the majority of Rho GTPases in the cytoplasm, maintaining Rho in an inactive form and inducing the disruption of Rho-dependent cell motility $(24,25)$. By contrast, RhoGDI2 acted as an escort protein directing Rho GTPases to the membrane and associated with active forms of Rho, Rac and cdc42, maintaining Rho in an active form $(26,27)$. Our results indicate that knockdown of RhoGDI2 expression in PC cells resulted in decreased cell motility and decreased invasion ability. However, the manner in which RhoGDI2 impacts on the activation of Rho GTPases requires further analysis. In addition to Rho GTPases activities, other mechanisms of RhoGDI2 in cancer have been reported in some studies. $\beta 1$-integrin and cyclooxygenase- 2 , which play key roles in breast cancer progression, were identified as target genes of RhoGDI2 in breast cancer cells $(12,28)$. PLC $\gamma$ were validated to be required for RhoGDI2-mediated cell invasion and metastasis in gastric cancer cells (29).

Tumor invasion and metastasis are the critical characteristics in determining the aggressive phenotype of human cancers, and require both tumor cell migration and degradation of matrix barriers (30). MMPs are involved in the degradation of ECM and basement membranes. Binker et al found that activation of Rac1 in PANC-1 cells were responsible for MMP2 secretion and activation (31). In the present study, we observed that the expression of MMP2 was significantly decreased following depletion of RhoGDI2 in PANC-1 and Patu8988 cells. Additionally, we found that MMP2 was positively correlated with RhoGDI2 expression in clinical PC tissues at the mRNA and protein levels. Thus, RhoGDI2 contributes to PC cell motility and invasion, at least in part by activating MMP2.

In summary, results of the present study demonstrated that the overexpression of RhoGDI2 was associated with PC progression. Depletion of RhoGDI2 in PC cells inhibited cell motility and invasion in vitro, at least in part by affecting the expression of MMP2. Our findings indicate that targeting RhoGDI 2 by a genetic approach may provide a new strategy for the treatment in PC.

\section{Acknowledgements}

This study was supported by Project of Nature Science Foundation of China (81201905), China Postdoctoral Science Foundation (2013M540374), Shanghai Postdoctoral Scientific Program of China (13R21415200) and Project of Medical Research of Jiangsu Province (H201209).

\section{References}

1. Siegel R, Naishadham D and Jemal A: Cancer statistics. CA Cancer J Clin 62: 10-29, 2012.

2. Vincent A, Herman J, Schulick R, Hruban RH and Goggins M: Pancreatic cancer. Lancet 378: 607-620, 2011.

3. Dai H, Li R, Wheeler T, et al: Enhanced survival in perineural invasion of pancreatic cancer: an in vitro approach. Hum Pathol 38: 299-307, 2007.

4. Kleeff J, Beckhove P, Esposito I, et al: Pancreatic cancer microenvironment. Int J Cancer 121: 699-705, 2007.

5. Vega FM and Ridley AJ: Rho GTPases in cancer cell biology. FEBS Lett 582: 2093-2101, 2008.

6. Reymond N, Riou P and Ridley AJ: Rho GTPases and cancer cell transendothelial migration. Methods Mol Biol 827: 123-142, 2012.

7. Garcia-Mata R, Boulter E and Burridge K: The 'invisible hand': regulation of RHO GTPases by RHOGDIs. Nat Rev Mol Cell Biol 12: 493-504, 2011.

8. Scherle P, Behrens T and Staudt LM: Ly-GDI, a GDP-dissociation inhibitor of the RhoA GTP-binding protein, is expressed preferentially in lymphocytes. Proc Natl Acad Sci USA 90: 7568-7572, 1993.

9. Cho HJ, Baek KE and Yoo J: RhoGDI2 as a therapeutic target in cancer. Expert Opin Ther Targets 14: 67-75, 2010.

10. Cho HJ, Baek KE, Park SM, et al: RhoGDI2 expression is associated with tumor growth and malignant progression of gastric cancer. Clin Cancer Res 15: 2612-2619, 2009.

11. Li X, Wang J, Zhang X, Zeng Y, Liang L and Ding Y: Overexpression of RhoGDI2 correlates with tumor progression and poor prognosis in colorectal carcinoma. Ann Surg Oncol 19: 145-153, 2012.

12. Zhang Y and Zhang B: D4-GDI, a Rho GTPase regulator, promotes breast cancer cell invasiveness. Cancer Res 66: 5592-5598, 2006.

13. Gildea JJ, Seraj MJ, Oxford G, et al: RhoGDI2 is an invasion and metastasis suppressor gene in human cancer. Cancer Res 62: 6418-6423, 2002.

14. Ma L, Xu G, Sotnikova A, et al: Loss of expression of LyGDI (ARHGDIB), a rho GDP-dissociation inhibitor, in Hodgkin lymphoma. Br J Haematol 139: 217-223, 2007.

15. Dransart E, Olofsson B and Cherfils J: RhoGDIs revisited: novel roles in Rho regulation. Traffic 6: 957-966, 2005. 
16. Boulter E, Garcia-Mata R, Guilluy C, et al: Regulation of Rho GTPase crosstalk, degradation and activity by RhoGDI1. Nat Cell Biol 12: 477-483, 2010.

17. Morin A, Cordelières FP, Cherfils $\mathrm{J}$ and Olofsson B: RhoGDI3 and RhoG: vesicular trafficking and interactions with the Sec3 exocyst subunit. Small GTPases 1: 142-156, 2010.

18. Lelias JM, Adra CN, Wulf GM, et al: cDNA cloning of a human mRNA preferentially expressed in hematopoietic cells and with homology to a GDP-dissociation inhibitor for the rho GTP-binding proteins. Proc Natl Acad Sci USA 90: 1479-1483, 1993.

19. Boulter E and Garcia-Mata R: RhoGDI: A rheostat for the Rho switch. Small GTPases 1: 65-68, 2010.

20. Harding MA and Theodorescu D: RhoGDI signaling provides targets for cancer therapy. Eur J Cancer 46: 1252-1259, 2010

21. Niu H, Li H, Xu C and He P: Expression profile of RhoGDI2 in lung cancers and role of RhoGDI2 in lung cancer metastasis. Oncol Rep 24: 465-471, 2010.

22. Cho HJ, Baek KE, Kim IK, et al: Proteomics-based strategy to delineate the molecular mechanisms of RhoGDI2-induced metastasis and drug resistance in gastric cancer. J Proteome Res 11: 2355-2364, 2012.

23. Theodorescu D, Sapinoso LM, Conaway MR, Oxford G, Hampton GM and Frierson HF Jr: Reduced expression of metastasis suppressor RhoGDI2 is associated with decreased survival for patients with bladder cancer. Clin Cancer Res 10: 3800-3806, 2004.
24. Dovas A and Couchman JR: RhoGDI: multiple functions in the regulation of Rho family GTPase activities. Biochem J 390: 1-9, 2005.

25. DerMardirossian C and Bokoch GM: GDIs: central regulatory molecules in Rho GTPase activation. Trends Cell Biol 15: 356-363, 2005.

26. Hart MJ, Maru Y,Leonard D, Witte ON, Evans T and Cerione RA: A GDP dissociation inhibitor that serves as a GTPase inhibitor for the Ras-like protein CDC42Hs. Science 258: 812-815, 1992.

27. Chuang TH, Xu X, Knaus UG, Hart MJ and Bokoch GM: GDP dissociation inhibitor prevents intrinsic and GTPase activating protein-stimulated GTP hydrolysis by the Rac GTP-binding protein. J Biol Chem 268: 775-778, 1993.

28. Schunke D, Span P, Ronneburg H, et al: Cyclooxygenase-2 is a target gene of rho GDP dissociation inhibitor beta in breast cancer cells. Cancer Res 67: 10694-10702, 2007.

29. Cho HJ, Baek KE, Nam IK, et al: PLC $\gamma$ is required for RhoGDI2mediated cisplatin resistance in gastric cancer. Biochem Biophys Res Commun 414: 575-580, 2011.

30. Friedl $\mathrm{P}$ and Wolf K: Tumour-cell invasion and migration: diversity and escape mechanisms. Nat Rev Cancer 3: 362-374, 2003.

31. Binker MG, Binker-Cosen AA, Richards D, Oliver B and CosenBinker LI: EGF promotes invasion by PANC-1 cells through Rac1/ROS-dependent secretion and activation of MMP-2. Biochem Biophys Res Commun 379: 445-450, 2009. 